Journal Club

Editor's Note: These short, critical reviews of recent papers in the Journal, written exclusively by graduate students or postdoctoral fellows, are intended to summarize the important findings of the paper and provide additional insight and commentary. For more information on the format and purpose of the Journal Club, please see http://www.jneurosci.org/misc/ifa_features.shtml.

\title{
The Hippocampus Contributes to Allocentric Spatial Memory through Coherent Scene Representations
}

\author{
Celia Fidalgo and Chris B. Martin \\ Department of Psychology, University of Toronto St. George, Toronto, Ontario M5S 1A1, Canada \\ Review of Guderian et al.
}

The ability to encode and retrieve the spatial relationships among elements of our environment is one way in which episodic memory supports adaptive behavior. Allocentric spatial memory refers specifically to mnemonic representations that capture viewpoint-invariant relations among items, as well as fixed relations between items and the local environment. Historically, the hippocampus has figured prominently in models of allocentric spatial memory. However, results from recent studies highlight potentially important contributions of subcortical regions in supporting spatial representations as well. In this context, lesion research and single-unit recording studies in rodents have identified an interesting functional distinction. Whereas the hippocampus contributes to memory for object location relative to spatial boundaries (i.e., boundary-based), the dorsal striatum contributes to memory for object location relative to landmarks or static placeholders in the environment (i.e., landmark-based; McDonald and White, 1994; Packard and McGaugh, 1996). This pattern of results has been corroborated by evidence obtained using fMRI in healthy humans (Doeller et al., 2008). As landmarks are typically stationary

Received Dec. 20, 2015; revised Jan. 21, 2016; accepted Jan. 25, 2016.

We thank Dr. Morgan Barense and Dr. Andy Lee for their continuous support.

Correspondence should be addressed to either Celia Fidalgo or Chris Martin, Department of Psychology, University of Toronto St. George, 27 King's College Circle, Toronto, 0N M5S 1A1, Canada. E-mail: celia.fidalgo@mail.utoronto.ca or cmarti97@gmail.com.

DOI:10.1523/JNEUROSCI.4548-15.2016

Copyright $\odot 2016$ the authors $\quad 0270-6474 / 16 / 362555-03 \$ 15.00 / 0$ objects, these findings suggest dorsal striatal involvement in processing object-object spatial relationships, a function generally attributed to the hippocampus in widely accepted theories of memory (Eichenbaum et al., 1994; Ranganath, 2010). However, whether hippocampal and dorsal striatal contributions are necessary and sufficient in supporting boundary-based and landmarkbased allocentric spatial memory, respectively, remains unclear.

In a recent issue of The Journal of Neuroscience, Guderian et al. (2015) took a significant step toward addressing this issue as it relates to the hippocampus. The authors used a virtual navigation task designed to probe allocentric spatial memory in two groups of patients with hippocampal damage. Individuals characterized as having 14.6-25.8\% volume reduction $(N=13)$ comprised the moderate hippocampal damage (MHD) group and those with $30.4-66.8 \%$ volume reduction $(N=15)$ comprised the severe hippocampal damage (SHD) group. Throughout the task, participants freely navigated an outdoor virtual environment presented on a computer screen. Importantly, distal cues such as clouds and mountains allowed participants to orient themselves in the environment and were present across all experimental conditions. During an initial learning phase, the environment was enclosed by a circular boundary and contained a single landmark object. Participants were instructed to learn the locations of four additional objects in the virtual environment. At test, participants were cued with a target object and instructed to navigate to the studied location of that object relative to either the boundary presented in the absence of the landmark (i.e., boundary-only) or the landmark presented in the absence of the boundary (i.e., landmark-only). Memory errors were quantified as the Euclidean distance between the selected location and the target location. To test the empirically derived prediction that the hippocampus differentially contributes to boundary-based allocentric spatial memory, structural volumetric assessments were used to predict memory errors on boundary-only and landmark-only trials. In addition to the hippocampus, the authors also explored the relationship between memory errors and volume of the dorsal striatum (caudate nucleus and putamen), thalamus, pallidum, and nucleus accumbens.

In line with evidence from previous research linking the hippocampus to boundary-based spatial memory (McDonald and White, 1994; Packard and McGaugh, 1996; Doeller et al., 2008), an initial cluster analysis revealed that error distance during boundary-based navigation was qualitatively better at distinguishing SHD from both MHD and controls than was error distance during landmark-based navigation. Importantly, however, results from two separate regression analyses revealed that, of the regions examined, only hippocampal volume predicted boundary- and landmark-based spatial memory errors. These results suggest that the hippocampus plays a role in 


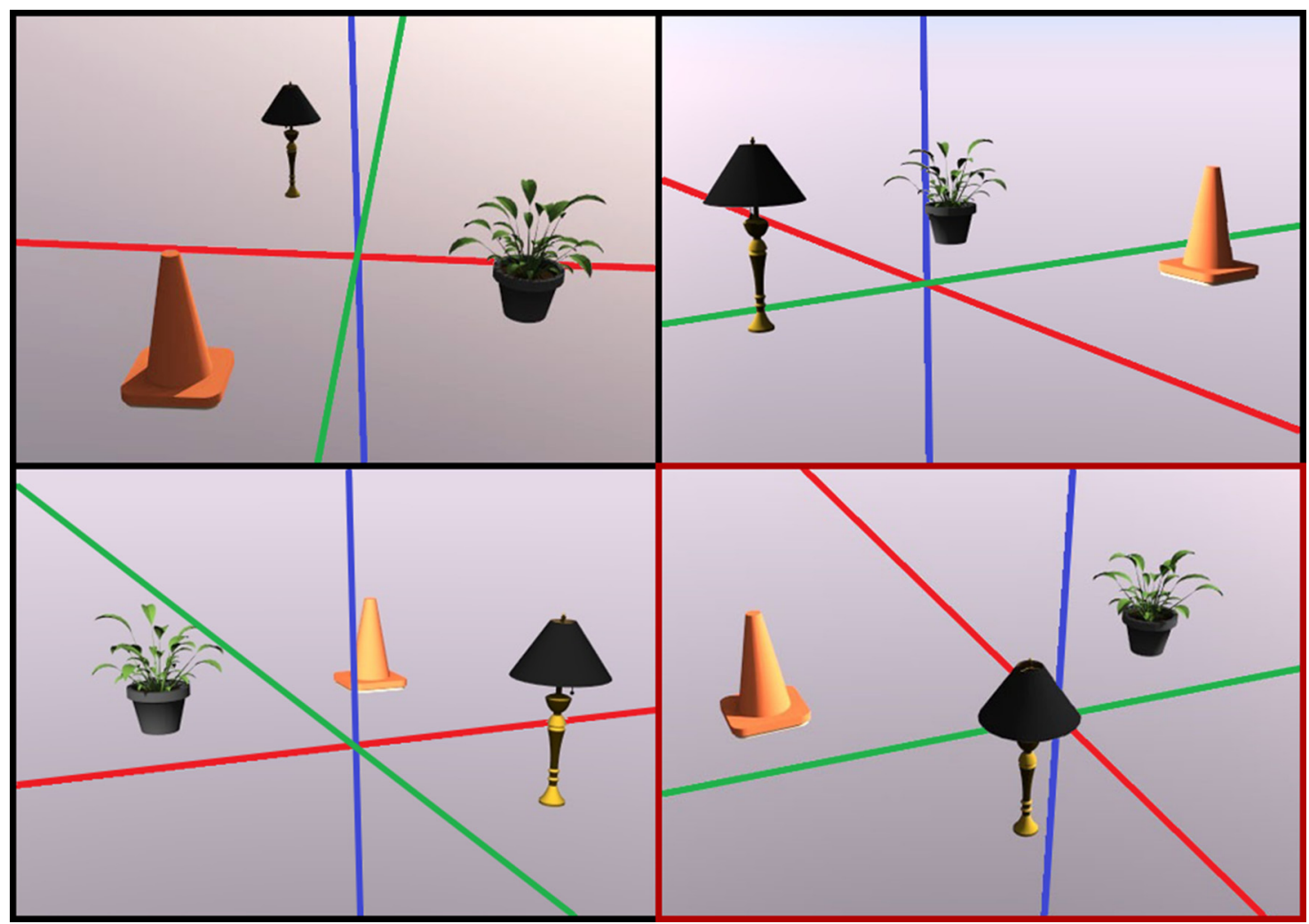

Figure 1. Sample trial from an oddball detection task. Participants must choose which of the four images depicts a unique spatial configuration of three objects. Three of the images depict identical spatial arrays of three objects (i.e., all objects are in the same location) taken from different viewpoints. The fourth image, outlined in red, depicts a unique configuration of the objects, as two (plant and lamp) have swapped positions. $x$-, $y$-, and $z$-axes have been included for demonstrative purposes only. Image created using Google SketchUp 2014.

both types of allocentric spatial memory, a finding at odds with extant fMRI evidence linking the hippocampus to boundarybased, but not landmark-based, allocentric spatial memory (Doeller et al., 2008). Although Guderian et al. (2015) focused their discussion on reconciling this apparent discrepancy, a theoretical framework that emphasizes representational demands across memory and perception (Lee et al., 2012) was not considered.

Representational accounts ascribe to the hippocampus a fundamental role in representing scenes and spatial expanses (Lee et al., 2005; Mullally and Maguire, 2013; Zeidman et al., 2014). Specifically, the hippocampus is thought to support viewpoint-invariant representations of scenes and the elements that define them, including geographical boundaries such as walls, ground, and ceiling. This view further suggests that hippocampal dysfunction affects scene representations most severely, and that allocentric relations between non-scene stimuli (e.g., between-object relations) may be somewhat preserved. As it relates to the study by Guderian et al. (2015), this perspective suggests that patients with hippocampal damage may indeed have greater difficulty representing boundary-based informa- tion than landmark-based information, as boundaries are necessary to define a spatial structure. Indeed, this line of reasoning is consistent with previous fMRI work (Doeller et al., 2008) and analogous to the hypothesis tested by Guderian et al. (2015). Importantly, a representational framework provides an interesting and previously unexplored explanation for the apparent discrepancy between fMRI results reported by Doeller et al. (2008) and neuropsychological evidence from Guderian et al. (2015). With respect to the latter, the landmark-based allocentric spatial memory deficits revealed in patients with SHD may reflect a fundamental inability to faithfully represent the virtual environment, and all spatially relevant cues, in a coherent manner.

As previously noted, although landmark-only trials used by Guderian et al. (2015) did not include the highly salient circular boundary, objects were still presented in a scene context (i.e., in the presence of distal cues) that provided a broader spatial layout. Consequently, it is difficult to rule out the possibility that the patients' inability to accurately represent the orienting distal cues and scene expanse of the virtual environment was a factor underlying task performance in both experimental conditions. Moreover, these scene elements may have provided a meaningful frame of reference that helped define the spatial relationships between objects. Accordingly, the extent to which performance on landmark-only trials reflected a process-pure measure of the spatial relationships between objects, and not the relationships between objects relative to additional non-boundary spatial cues, remains unclear. With this caveat in mind, it is possible that the domain-general allocentric spatial memory impairments reported for SHD patients in Guderian et al. (2015) reflect a fundamental inability to faithfully represent scenes and their spatial geometry, rather than a deficit in both boundarybased and landmark-based memory. The theoretical importance of this point is highlighted by previous fMRI and neuropsychological research that has linked hippocampal damage to impoverished scene representations (for review, see Graham et al., 2010).

One way in which a representational framework may aid in demonstrating differential hippocampal involvement in boundary-based allocentric processing is in regards to the cognitive domain probed by the task. Whereas Guderian et al. 
(2015) were theoretically motivated to examine spatial processing using a memoryguided navigation task, representational frameworks posit that hippocampus-dependent representations support both mnemonic and perceptual discriminations, suggesting that perceptual tasks may also be effective in examining allocentric processing. This is particularly relevant in relation to patients in the SHD group, who did not appear to learn the locations of objects across encoding blocks. Performance during the initial learning phase in this group was significantly impaired relative to both MHD patients and healthy controls (see Guderian et al., 2015, their Fig. 5). Thus, one promising alternative approach to examine whether hippocampal damage differentially affects boundary- and landmark-based allocentric spatial relationships is through the use of a visual perception task, such as oddball detection. Such a task could involve concurrent presentation of four object arrays, each of which comprise identical objects without additional scene elements. Importantly, three of these arrays could depict identical spatial configurations of the objects (i.e., all objects presented in the exact same position) from different viewpoints. The fourth array includes the same objects presented in a novel spatial arrangement (i.e., the oddball; see Fig. 1). On each trial, the participant is tasked with identifying the configural oddball. Although this particular task does not assess memory-guided navigation performance, the general stimulus design could be adapted for use in a mnemonic navigational task, with the crucial element being the absence of scenespecific geometry. Intact performance by patients with SHD on such tasks would be consistent with the notion that structures beyond the hippocampus, such as dorsal striatal regions, support representations of spatial relationships among objects, and additionally demonstrate that allocentric spatial processing in the hippocampus is not absolute. Comparing performance of patients on the ecologically valid task used by Guderian et al. (2015) to that of a task that permits a more direct process-pure inference may provide a clearer picture of differential contributions of the hippocampus to allocentric spatial representations.

In summary, Guderian et al. (2015) present important work that extend our understanding of the relative contributions of the hippocampus and subcortical regions to allocentric spatial processing. Their findings point toward a need to dissociate object-based and scene-based allocentric processing, a distinction that will help guide future endeavors.

\section{References}

Doeller CF, King JA, Burgess N (2008) Parallel striatal and hippocampal systems for landmarks and boundaries in spatial memory. Proc Natl Acad Sci U S A 105:5915-5920. CrossRef Medline

Eichenbaum H, Otto T, Cohen NJ (1994) Two functional components of the hippocampal memory system. Behav Brain Sci 17:449-472. CrossRef

Graham KS, Barense MD, Lee AC (2010) Going beyond LTM in the MTL: a synthesis of neu- ropsychological and neuroimaging findings on the role of the medial temporal lobe in memory and perception. Neuropsychologia 48:831-853. CrossRef Medline

Guderian S, Dzieciol AM, Gadian DG, Jentschke S, Doeller CF, Burgess N, Mishkin M, VarghaKhadem F (2015) Hippocampal volume reduction in humans predicts impaired allocentric spatial memory in virtual-reality navigation. J Neurosci 35:14123-14131. CrossRef Medline

Lee AC, Bussey TJ, Murray EA, Saksida LM, Epstein RA, Kapur N, Hodges JR, Graham KS (2005) Perceptual deficits in amnesia: challenging the medial temporal lobe "mnemonic" view. Neuropsychologia 43:1-11. CrossRef Medline

Lee AC, Yeung LK, Barense MD (2012) The hippocampus and visual perception. Front Hum Neurosci 6:91. CrossRef Medline

McDonald RJ, White NM (1994) Parallel information processing in the water maze: evidence for independent memory systems involving dorsal striatum and hippocampus. Behav Neural Biol 61:260-270. CrossRef Medline

Mullally SL, Maguire EA (2013) Memory, imagination, and predicting the future: a common brain mechanism? Neuroscientist 20:220234. CrossRef Medline

Packard MG, McGaugh JL (1996) Inactivation of hippocampus or caudate nucleus with lidocaine differentially affects expression of place and response learning. Neurobiol Learn Mem 65:65-72. CrossRef Medline

Ranganath C (2010) A unified framework for the functional organization of the medial temporal lobes and the phenomenology of episodic memory. Hippocampus 20:1263-1290. CrossRef Medline

Zeidman P, Mullally SL, Maguire EA (2015) Constructing, perceiving, and maintaining scenes: hippocampal activity and connectivity. Cereb Cortex 25:3836-3855. CrossRef Medline 